\title{
EFEITO DO TEMPO DE FORNECIMENTO DE RAÇÃO SUPLEMENTADA COM ÓLEO DE LINHAÇA SOBRE A COMPOSIÇÃO FÍSICO-QUÍMICA E DE ÁCIDOS GRAXOS EM CABEÇAS DE TILÁPIAS DO NILO (Oreochromis niloticus) ${ }^{1}$
}

\author{
Jesuí V. VISENTAINER², Sandra T. M. GOMES², Carmino HAYASHI², Oscar O. SANTOS-JÚNIOR²,
}

\author{
Adriano B. M. da SILVA ${ }^{2}$, Karin C. JUSTI ${ }^{2}$, Nilson E. de SOUZA², Makoto MATSUSHITA ${ }^{2, *}$
}

\section{RESUMO}

Este trabalho teve como objetivo realizar um estudo sobre a composição físico-química e, especialmente sobre a composição de ácidos graxos nos lipídios totais de cabeças de tilápias (Oreochromis niloticus), submetidas a diferentes tempos $(0,10,20,30$ dias) de fornecimento de ração suplementada com óleo de linhaça (fonte do ácido $\alpha$-linolênico, 18:3n-3 LNA), em substituição ao óleo de girassol. Não houveram diferenças $(\mathrm{P}>0,05)$ entre os teores de umidade, cinza, proteina e lipídios totais para os diferentes tratamentos (tempos de fornecimento). Os teores de lipídios totais das cabeças variaram de 8,41 a 10,13\% e foi encontrado um total de 27 ácidos graxos nestes lipídios para todos os tratamentos. Os ácidos graxos majoritários foram 18:2n-6 (29,66 a 32,11\%), 18:1n-9 (29,68 a 31,71\%) e $16: 0$ $(15,53$ a $16,63 \%)$. Houve um aumento nos teores percentuais do ácido 18:3n-3, da somatória de ácidos graxos ômega-3 e uma diminuição da razão n-6/n-3, com diferenças $(\mathrm{P}<0,05)$ entre o tratamento A (tempo zero) e os demais tratamentos que receberam óleo de linhaça. Não houveram diferenças $(\mathrm{P}>0,05)$ entre os tratamentos para a razão entre ácidos graxos polinsaturados e saturados (AGPI/AGS).

Palavras-chave: Tilápia do Nilo (Oreochromis niloticus); cabeça; ácidos graxos; ômega-3; óleo de linhaça.

\section{SUMMARY}

EFFECT OF RATION SUPPLY TIME WITH FLAXSEED OIL ON PHYSICO-CHEMICAL COMPOSITION AND FATTY ACIDS OF NILE TILAPIA (Oreochromis niloticus) HEADS. The main of this study was to analyze the physical-chemical composition and, especially the fatty acids composition, in the total lipids of tilapia (Oreochromis niloticus) heads, submitted to different ration supply times $(0,10,20,30$ days) with flaxseed oil (source of $\alpha$-linolenic acid 18:3 n-3, LNA), in substitution to the sunflower oil. There were not significant differences among $(P>0,05)$ the moisture, ash, protein, and total lipids values for the different treatments (times of supply). The values of total lipids found in the heads varied from 8.41 to $10.13 \%$, and 27 fatty acids were found in these lipids in all treatments. The majority fatty acids were 18:2n-6, 18:1n-9 and 16:0. There was an increase in the LNA acid percentage values, in the omega-3 (n-3) sum, and a decrease of the $n-$ 6/n-3 reason, with significant differences among the treatment A (zero time) and other treatments that received flaxseed oil. There were not significant differences among the treatments in relation to polyunsaturated fatty acids/saturated fatty acids (PUFA/SFA) rate. Keywords: Nile Tilapia ((Oreochromis niloticus); heads; fatty acids; omega-3; flaxseed oil.

\section{1 - INTRODUÇÃO}

O uso de ácidos graxos, óleos de peixes como suplemento e peixes na alimentação humana têm sido objeto de inúmeras pesquisas nos últimos anos. Foram evidenciados os efeitos benéficos dos ácidos graxos polinsaturados (AGPI) de pescado nas populações que residem em área de pesca [14].

Tem sido atribuída uma elevada importância nutricional aos ácidos da família ômega-3 (ou n-3), especialmente aos ácidos alfa-linolênico 18:3n-3 (LNA), eicosapentaenóico 20:5n-3 (EPA) e docosa-hexaenóico 22:6n-3 (DHA). Assim, inúmeras pesquisas surgiram sobre os ácidos graxos ômega-3 e, conseqüentemente, as indústrias alimenticias e farmacêuticas suplementaram alimentos e desenvolveram concentrados com ômega-3 em todo o mundo.

Os resultados das pesquisas vêm confirmando que um aumento na ingestão de AGPI n-3 reduz a taxa total de colesterol no sangue. Além disso, estudos realizados com base em intervenções de dietas comprovaram que o consumo de AGPI e/ou óleos de pescado reduz fatores bioquímicos de risco associados a doenças 1. Recebido para publicação em 20/09/2002. Aceito para publicação em 17/02/2003 (000958).

${ }^{2}$ Departamento de Química, Universidade Estadual de Maringá $A v$. Colombo, $5790-C E P-87020-900$ - Maringá - PR

* A quem a correspondência deve ser enviada. cardiovasculares [9,27], psoriase [18], artrite e câncer $[22,24,31]$ e pode estar envolvido na fertilidade humana [5].

Pesquisadores acreditam que, atualmente as dietas de ácidos graxos ômega-3 e ômega-6 nos ocidentais apresentam razão n-6/n-3 de aproximadamente 20 a 30:1, valores muito elevados quando comparados com aqueles considerados ideais de 1 a 2:1 [25]. Os elevados valores da razão n-6/n-3 geraram um desbalanceamento de ácidos graxos no organismo humano e, provavelmente, contribuiram para o desenvolvimento de processos inflamatórios, desordem do sistema imune, hipertensão e disfunções neurológicas [14].

Os ácidos alfa-linolênico 18:3n-3 (LNA) e linoléico 18:2n-6 (LA) são considerados ácidos graxos essenciais e precursores dos demais ácidos da família n-3 e n-6, respectivamente. $\mathrm{O}$ ácido LA pode ser encontrado em abundância nos óleos de milho, girassol, soja, dentre outros. Enquanto, o ácido LNA é encontrado em concentrações elevadas na semente de linho (Linum usitatissimum), a qual apresenta 32 a $38 \%$ de óleo e teores percentuais de LNA que variam de 44,6 a 51,5\% do total dos ácidos graxos [3,4].

No Brasil, nos últimos anos, ocorreu e continua ocorrendo um crescimento vertiginoso na criação e consumo de peixes em cativeiro. Isto ocorre devido a condições hidrográficas favoráveis, ao crescimento de "pesque e 
pague", "pague e pesque", de novas espécies de peixes e ao fornecimento destes peixes pelos produtores, os quais vendem seus produtos em mercados, pesqueiros, feiras livres, etc. Neste crescimento, destaca-se principalmente espécies como a tilápia (Oreochromis niloticus) que apresenta uma boa aceitação pelo consumidor.

As tilápias (Oreochromis niloticus) são espécies exóticas cultivadas no Brasil desde 1971. Esta espécie apresenta no seu tecido muscular, baixos teores dos ácidos LNA, EPA e DHA, e outros ácidos graxos da família n-3 [15].

Os resíduos dos peixes obtidos nos processos de filetagem ou de outros processos incluem a cabeça, a pele e as vísceras. As cabeças de peixes não apresentam uma finalidade definida pelos produtores, comumente são utilizadas na fabricação de farinha de peixe juntamente com outros resíduos, porém, devido a dificuldades de transporte e/ou pelo baixo preço, elas são enterradas para evitar a contaminação dos tanques. Isto ocorre freqüentemente nos pesqueiros. Algumas vezes, as cabeças são doadas para entidades assistenciais ou consumidores para serem utilizadas na alimentação sob a forma de sopas, caldos, pirão, assadas, etc.

Estudos sobre a composição de ácidos graxos nos lipídios da cabeça de peixes começaram a surgir no Brasil nos últimos anos. Provavelmente, trabalhos pioneiros que estudaram parte da cabeça de peixes, particularmente a composição dos olhos ou da cavidade ocular foram realizados por VISENTAINER et al.[28] e SILVA [23].

O objetivo deste trabalho foi avaliar a composição físico-química de cabeças de tilápias alimentadas com óleo de linhaça, especialmente a composição em ácidos graxos dos lipídios e, ainda, obter informações sobre a transferência de ácidos graxos ômega-3 da ração com óleo de linhaça para o conteúdo lipídico das cabeças, uma vez que, estudos desta natureza são inexistentes no Brasil.

\section{2 - MATERIAL E MÉTODOS.}

\section{1 - Amostragem e rações}

Na estação de piscicultura do Departamento de Biologia da Universidade Estadual de Maringá - PR, alevinos de tilápia (Oreochromis niloticus), foram submetidos a um sistema de confinamento. Em 20 tanques com capacidade de 1000L/cada, com sistema de sifonagem e borbulhamento de ar atmosférico, foram colocados 5 exemplares de alevinos/caixa.

Os alevinos de tilápias, com peso médio 41,66 $\pm 0,56 \mathrm{~g}$, foram submetidos a um experimento completamente aleatorizado com 4 tratamentos e 5 repetições. Os tratamentos consistiram no fornecimento de dietas (Tabela 1) enriquecidas com óleo de linhaça (em substituição ao óleo de girassol) por diferentes períodos: 0, 10, 20 e 30 dias de fornecimento (tratamentos A, B, C e D, respectivamente).

Todos os ingredientes das rações I e II foram comuns em quantidade e qualidade, exceto na complementação dos óleos adicionados, conforme mostra a Tabela 1.
TABELA 1. Composição percentual em massa dos ingredientes rações I e II.

\begin{tabular}{lcc}
\hline \multicolumn{1}{c}{ INGREDIENTES } & RAÇãO I (óleo de girassol) & RAÇÃO II (óleo de linhaça) \\
\hline \% Farelo de Milho & 16,93 & 16,93 \\
\% Farelo de Soja & 51,62 & 51,62 \\
\% Farelo de Trigo & 20,00 & 20,00 \\
\% Bagaço de Cana & 1,28 & 1,28 \\
\% Calcário & 1,74 & 1,74 \\
\% Fosfato Bicálcico & 2,41 & 2,41 \\
\% Óleo de Lin haça & $\mathbf{0 , 0 0}$ & $\mathbf{3 , 7 5}$ \\
\% Óleo de Girassol & $\mathbf{5 , 0 0}$ & $\mathbf{1 , 2 5}$ \\
\% BHT* & 0,02 & 0,02 \\
\% Sal (NaCl) & 0,50 & 0,50 \\
\% Premix** & 0,50 & 0,50 \\
\hline
\end{tabular}

*Butil-hidróxi-tolueno (antioxidante)

Negrito $=\%$ de óleo de linhaça e girassol

Foram realizadas análises teor de umidade, cinza, proteina bruta, lipídios totais e composição de ácidos graxos nos lipídios totais das amostras de cabeça de tilápia. Nas rações I e II foram analisadas a composição de ácidos graxos nos lipídios totais. Todas as análises foram realizadas em triplicatas.

\section{2 - Umidade e cinza}

As análises de umidade e cinza foram realizadas conforme técnicas da AOAC de acordo com CUNNIFF [6].

\section{3 - Proteina bruta}

A análise do teor de proteína bruta foi baseada no processo semi-micro Kjeldahl, conforme técnicas da AOAC [6].

\section{4 - Extração e teor de lipídios totais}

Na extração dos lipídios totais foi empregado o método de BLIGH \& DYER [2], considerando-se as proporções recomendadas entre os solventes: metanol, clorofórmio e água tissular.

A determinação quantitativa dos lipídios totais foi realizada gravimetricamente, com modificações do método de MAIA [15], eliminando-se o clorofórmio (fração clorofórmio-lipídios) em evaporador rotatório a vácuo, com banho a $30^{\circ} \mathrm{C}$ e o residuo remanescente de solventes eliminado com fluxo de nitrogênio.

\section{5 - Transesterificação dos lipídios totais}

Os lipidios totais foram submetidos ao processo de transesterificação para a preparação dos ésteres metílicos de ácidos graxos, conforme método 5509 da ISO [12]. A fase superior (n-heptano e ésteres metílicos de ácidos graxos) foi transferida para frascos de $5 \mathrm{~mL}$ de capacidade, fechados hermeticamente e armazenados em congelador $\left(-18^{\circ} \mathrm{C}\right)$, para posterior análise cromatográfica. 


\section{6 - Análise cromatográfica dos ésteres metílicos de ácidos graxos}

Os ésteres de ácidos graxos foram analisados em um cromatógrafo gasoso 14-A (Shimadzu, Japão), equipado com coluna capilar de sílica fundida $(50 \mathrm{~m}$ de comprimento, $0,25 \mathrm{~mm}$ de diâmetro interno e $0,20 \mu \mathrm{m}$ de Carbowax 20M) e detector de ionização de chama. Os fluxos dos gases foram de 1,2mL. min $^{-1}$ para o gás de arraste $\mathrm{H}_{2}, 30 \mathrm{~mL} \cdot \mathrm{min}^{-1}$ para o gás auxiliar ("make-up") $\mathrm{N}_{2}$ e 30 e $300 \mathrm{~mL} \cdot \mathrm{min}^{-1}$ para os gases da chama $\mathrm{H}_{2}$ e ar sintético, respectivamente. A razão de divisão ("split") da amostra foi de 1:100. A temperatura da coluna foi programada a $150^{\circ} \mathrm{C}$ por 5 minutos, sendo então elevada para $240^{\circ} \mathrm{C}$ a uma taxa de $2^{\circ} \mathrm{C} \cdot \mathrm{min}^{-1}$. As temperaturas do injetor e detector foram $220^{\circ} \mathrm{C}$ e $245^{\circ} \mathrm{C}$, respectivamente. As injeções foram realizadas em triplicatas e o volume de injeção foi de $1 \mu \mathrm{L}$. As áreas dos picos foram determinadas pelo método da normalização, utilizandose um Integrador-Processador CG-300 (Instrumentos Científicos CG), e a identificação dos picos foi feita por comparação dos tempos de retenção de padrões de ésteres metílicos de ácidos graxos.

\section{7 - Análise estatística}

Os resultados foram submetidos à análise de variância (ANOVA) a 5\% de probabilidade, pelo teste de Tukey, através do software Statistica, versão 5.0 [26].

\section{3 - RESULTADOS E DISCUSSÃO}

Após o abate, as tilápias apresentaram um valor médio de $151,5 \mathrm{~g}$ e as cabeças corresponderam a um rendimento percentual em massa de $31,4 \%$ em relação ao peso dos exemplares inteiros.

A Tabela 2 mostra a composição percentual de umidade, cinza, proteina e lipídios totais das cabeças de tilápias (Oreochromis niloticus) submetidas aos diferentes tratamentos (A, B, C e D) com as rações. Na composição físico-química das cabeças de tilápias não foram encontradas diferenças significativas entre os diferentes tratamentos em todas as análises realizadas.

TABELA 2. Composição físico-química das cabeças de tilápias (Oreochromis niloticus) submetidas aos diferentes tratamentos.

\begin{tabular}{lcccc}
\hline COMPOSIÇÃO & \multicolumn{3}{c}{ TRATAMENTOS (em número de dias) } \\
\cline { 2 - 5 } & $\mathrm{A}(0$ dias $)$ & $\mathrm{B}(10$ dias $)$ & $\mathrm{C}(20$ dias $)$ & $\mathrm{D}(30$ dias $)$ \\
\hline Umidade (\%) & $70,63^{\mathrm{a}} \pm 0,95$ & $69,71^{\mathrm{a}} \pm 1,45$ & $69,84^{\mathrm{a}} \pm 1,38$ & $70,44^{\mathrm{a}} \pm 2,52$ \\
Cinza (\%) & $4,76^{\mathrm{a}} \pm 0,85$ & $5,08^{\mathrm{a}} \pm 1,20$ & $4,93^{\mathrm{a}} \pm 1,09$ & $4,95^{\mathrm{a}} \pm 1,40$ \\
Lipídios totais (\%) & $8,41^{\mathrm{a}} \pm 0,82$ & $8,53^{\mathrm{a}} \pm 0,49$ & $10,13^{\mathrm{a}} \pm 0,35$ & $8,46^{\mathrm{a}} \pm 1,38$ \\
Proteína bruta (\%) & $14,06^{\mathrm{a}} \pm 1,41$ & $12,84^{\mathrm{a}} \pm 0,69$ & $12,59^{\mathrm{a}} \pm 0,79$ & $13,43^{\mathrm{a}} \pm 1,34$
\end{tabular}

Os resultados são médias com estimativas dos desvio padrão.

Letras diferentes na mesma linha indicam diferenças significativas $(p<0,05)$ pelo teste de Tukey.

VISENTAINER et al. [29] encontraram em cabeças de tilápias jovens, alimentadas com rações comerciais, teores percentuais de umidade de $73,15 \%$; cinza $3,72 \%$; lipídios totais $9,93 \%$ e proteina $10,65 \%$. Os valores da composição entre a cabeça das tilápias jovens e as cabeças das tilápias deste experimento (Tabela 2) mostraram composição variável, porém os valores apresentaram as mesmas tendências.

Comparando os valores de lipidios totais das cabeças dos diferentes tratamentos A $(8,41 \%)$, B $(8,53 \%)$, $\mathrm{C}(10,13 \%)$ e $\mathrm{D}(8,46 \%)$ das tilápias deste experimento (Tabela 2) com o teor de lipidios totais de 1,4\% [15] e $2,86 \%$ [1] em filés de tilápias (Oreochromis niloticus) adultas e com outras espécies de água doce, como o tucunaré $(0,8 \%)$ [23], corvina $(0,71)$, botoado $(2,50 \%)$ [20] e piranha $(0,70)[1]$, os valores de lipídios totais das cabeças dos diferentes tratamentos deste experimento foram muito superiores aos filés destes peixes.

Os valores elevados de lipidios totais das cabeças das tilápias deste experimento (Tabela 2), aliados ao rendimento percentual de $31,4 \%$ destas cabeças em relação ao peixe inteiro, fazem com que as cabeças sejam uma excelente fonte de lipidios totais.

A Tabela 3 mostra que, nas rações I (sem óleo de linhaça) e II (com óleo de linhaça) foram encontrados um total de 15 ácidos graxos.

TABELA 3. Composição percentual de ácidos graxos das rações I e II.

\begin{tabular}{|c|c|c|}
\hline Ácido Graxo & Ração I (sem óleo de linhaça) & Ração II (com óleo de linhaça) \\
\hline $14: 0$ & $0,24^{\mathrm{a}} \pm 0,01$ & $0,23^{\mathrm{a}} \pm 0,03$ \\
\hline $16: 0$ & $9,94^{\mathrm{a}} \pm 0,15$ & $9,26^{a} \pm 0,08$ \\
\hline $16: \ln -7$ & $0,30^{\mathrm{a}} \pm 0,01$ & $0,31^{\mathrm{a}} \pm 0,01$ \\
\hline $17: 0$ & $0,09^{\mathrm{a}} \pm 0,01$ & $0,10^{\mathrm{a}} \pm 0,00$ \\
\hline 18:0 & $4,06^{\mathrm{b}} \pm 0,10$ & $5,61^{a} \pm 0,07$ \\
\hline $18: \ln -9$ & $27,23^{\mathrm{b}} \pm 0,19$ & $24,59^{\mathrm{a}} \pm 0,47$ \\
\hline $18: \ln -7$ & $0,96^{\mathrm{b}} \pm 0,03$ & $1,61^{a} \pm 0,10$ \\
\hline $18: 2 \mathrm{n}-6$ & $53,83^{\mathrm{b}} \pm 0,21$ & $32,30^{\mathrm{a}} \pm 0,40$ \\
\hline $18: 3 n-3$ & $1,58^{\mathrm{h}} \pm 0,03$ & $24,24^{a} \pm 0,20$ \\
\hline 20:1n-11 & $0,42^{\mathrm{b}} \pm 0,00$ & $0,32^{\mathrm{a}} \pm 0,02$ \\
\hline $20: \ln -9$ & $0,26^{a} \pm 0,04$ & $0,38^{\mathrm{a}} \pm 0,01$ \\
\hline $20: 5 n-3$ & $0,07^{\mathrm{a}} \pm 0,02$ & $0,11^{\mathrm{a}} \pm 0,04$ \\
\hline $22: \ln -11$ & $0,66^{\mathrm{b}} \pm 0,04$ & $0,32^{\mathrm{a}} \pm 0,05$ \\
\hline $22: \ln -9$ & $0,15^{\mathrm{b}} \pm 0,01$ & $0,40^{\mathrm{a}} \pm 0,03$ \\
\hline $22: 6 n-3$ & $0,12^{\mathrm{b}} \pm 0,02$ & $0,28^{\mathrm{a}} \pm 0,03$ \\
\hline
\end{tabular}

Os resultados são médias com estimativas dos desvio padrão. Resultados expressos em percentagem do total de ácidos graxos Letras diferentes na mesma linha indicam diferenças significativas $(p<0,05)$ pelo
teste de Tukey.

Negrito = percentagem de 18:3n-3 nas diferentes rações.

A ração I (Tabela 3) apresentou teor de 1,58\% do ácido alfa-linolênico (18:3n-3), enquanto a ração II apresentou 24,24\%. Estas diferenças $(\mathrm{P}<0,05)$ são devido à composição dos óleos de linhaça e girassol adicionados. O óleo de linhaça apresenta elevada percentagem de 18:3n-3, enquanto o óleo de girassol apresenta percentagem baixa deste ácido. 
A percentagem do ácido linoléico (18:2n-6) foi elevada na ração I, devido aos elevados teores deste ácido no óleo de girassol, o qual foi adicionado nesta ração. A percentagem de 18:2n-6 na ração II foi inferior à ração I, devido à substituição parcial do óleo de girassol pelo óleo de linhaça, conforme mostra a Tabela 3.

Os resultados de análises da composição de ácidos graxos de diversas rações comerciais vendidas no Brasil mostraram os baixos teores (aproximadamente 3,3\%) do ácido 18:3n-3, um ácido graxo essencial e precursor de outros ácidos graxos da família n-3 de elevada importância nutricional. Nestas mesmas rações, foram encontrados elevados teores dos ácido 18:2 n-6 (aproximadamente $38,8 \%$ ), um ácido graxo também essencial e comumente encontrado em óleos vegetais como o de soja, milho, girassol, dentre outros [19].

A Tabela 4 mostra a composição percentual de ácidos graxos nos lipídios totais das cabeças das tilápias submetidas aos diferentes tratamentos com óleo de linhaça.

TABELA 4. Composição percentual em ácidos graxos das cabeças das tilápias submetidas aos diferentes tratamentos.

\begin{tabular}{|c|c|c|c|c|}
\hline \multirow[t]{2}{*}{ ÁCIDO GRAXO } & \multicolumn{4}{|c|}{ TRATAMENTOS (em número de dias) } \\
\hline & A ( 0 dias $)$ & B (10 dias) & C (20 dias) & $\mathrm{D}$ (30 dias) \\
\hline 14:0 & $0,77^{\mathrm{a}} \pm 0,08$ & $1,15^{\mathrm{ab}} \pm 0,15$ & $1,14^{\mathrm{ab}} \pm 0,08$ & $1,08^{\mathrm{a}} \pm 0,30$ \\
\hline 15:0 & $0,11^{\mathrm{a}} \pm 0,01$ & $0,11^{\mathrm{a}} \pm 0,09$ & $0,10^{\mathrm{a}} \pm 0,12$ & $0,12^{\mathrm{a}} \pm 0,03$ \\
\hline i - $16: 0$ & $0,19^{\mathrm{a}} \pm 0,01$ & $0,21^{\mathrm{a}} \pm 0,01$ & $0,22^{\mathrm{a}} \pm 0,06$ & $0,18^{\mathrm{a}} \pm 0,05$ \\
\hline 16:0 & $15,53^{\mathrm{a}} \pm 0,20$ & $16,24^{\mathrm{a}} \pm 0,52$ & $15,99^{\mathrm{a}} \pm 0,69$ & $16,63^{a} \pm 1,65$ \\
\hline $16: \ln -9$ & $0,47^{\mathrm{a}, \mathrm{c}} \pm 0,03$ & $0,52^{\mathrm{b}, \mathrm{c}} \pm 0,04$ & $0,57^{\mathrm{b}} \pm 0,09$ & $0,45^{\mathrm{a}} \pm 0,11$ \\
\hline $16: \ln -7$ & $1,77^{\mathrm{a}} \pm 0,08$ & $2,08^{\mathrm{a}} \pm 0,08$ & $2,22^{\mathrm{a}} \pm 0,11$ & $1,79^{\mathrm{a}} \pm 0,23$ \\
\hline$i-17: 0$ & $0,15^{\mathrm{a}} \pm 0,07$ & $0,18^{\mathrm{a}} \pm 0,03$ & $0,13^{\mathrm{a}} \pm 0,14$ & $0,19^{\mathrm{a}} \pm 0,31$ \\
\hline ai $-17: 0$ & $0,22^{\mathrm{a}} \pm 0,06$ & $0,22^{\mathrm{a}} \pm 0,03$ & $0,20^{\mathrm{a}} \pm 0,11$ & $0,27^{\mathrm{a}} \pm 0,19$ \\
\hline $17: 0$ & $0,32^{\mathrm{a}} \pm 0,02$ & $0,29^{\mathrm{a}} \pm 0,02$ & $0,30^{\mathrm{a}} \pm 0,02$ & $0,25^{\mathrm{a}} \pm 0,03$ \\
\hline $17: \ln -9$ & $0,14^{\mathrm{a}} \pm 0,02$ & $0,12^{\mathrm{a}} \pm 0,15$ & $0,12^{\mathrm{a}} \pm 0,14$ & $0,14^{a} \pm 0,03$ \\
\hline 18:0 & $5,72^{\mathrm{b}} \pm 0,36$ & $5,34^{\mathrm{ab}} \pm 0,75$ & $4,96^{\mathrm{a}} \pm 0,40$ & $4,90^{\mathrm{a}} \pm 0,32$ \\
\hline $18: \ln -9$ & $31,71^{\mathrm{a}} \pm 0,56$ & $29,68^{\mathrm{b}} \pm 0,67$ & $29,77^{\mathrm{b}} \pm 1,08$ & $31,62^{\mathrm{a}} \pm 1,32$ \\
\hline $18: \ln -7$ & $2,21^{\mathrm{a}} \pm 0,19$ & $2,15^{\mathrm{a}} \pm 0,19$ & $2,58^{\mathrm{a}} \pm 0,96$ & $2,25^{\mathrm{a}} \pm 0,21$ \\
\hline $18: 2 n-6$ & $32,11^{\mathrm{a}} \pm 0,86$ & $31,75^{\mathrm{a}} \pm 0,88$ & $30,48^{\mathrm{a}} \pm 0,40$ & $29,66^{\mathrm{a}} \pm 2,11$ \\
\hline $18: 3 n-6$ & $0,89^{\mathrm{a}} \pm 0,28$ & $1,10^{\mathrm{a}} \pm 0,39$ & $1,01^{\mathrm{a}} \pm 0,24$ & $0,81^{a} \pm 0,47$ \\
\hline $18: 3 n-3$ & $1,08^{\mathrm{c}} \pm 0,12$ & $2,04^{\mathrm{b}} \pm 0,31$ & $3,60^{\mathrm{a}} \pm 0,11$ & $3,80^{a} \pm 0,80$ \\
\hline $20: \ln -11$ & $0,34^{\mathrm{a}} \pm 0,06$ & $0,27^{\mathrm{b}} \pm 0,17$ & $0,28^{\mathrm{a} b} \pm 0,03$ & $0,28^{\mathrm{ab}} \pm 0,07$ \\
\hline $20: \ln -9$ & $1,23^{\mathrm{b}} \pm 0,11$ & $1,14^{\mathrm{b}} \pm 0,18$ & $1,14^{\mathrm{ab}} \pm 0,14$ & $0,99^{\mathrm{a}} \pm 0,14$ \\
\hline $20: 3 n-9$ & $1,63^{\mathrm{b}} \pm 0,50$ & $1,66^{\mathrm{b}} \pm 0,31$ & $1,48^{\mathrm{b}} \pm 0,16$ & $1,19^{\mathrm{a}} \pm 0,24$ \\
\hline 21:0 & $0,85^{\mathrm{b}} \pm 0,06$ & $0,91^{\mathrm{b}} \pm 0,29$ & $0,85^{\mathrm{ab}} \pm 0,26$ & $0,68^{\mathrm{a}} \pm 0,24$ \\
\hline $20: 4 n-6$ & $0,71^{\mathrm{ab}} \pm 0,06$ & $0,78^{\mathrm{b}} \pm 0,16$ & $0,73^{\mathrm{b}} \pm 0,29$ & $0,56^{a} \pm 0,16$ \\
\hline $20: 3 n-3$ & $0,21^{\mathrm{b}} \pm 0,02$ & $0,32^{\mathrm{b}} \pm 0,07$ & $0,53^{\mathrm{a}} \pm 0,09$ & $0,53^{a} \pm 0,08$ \\
\hline $22: \ln -11$ & $0,27^{\complement} \pm 0,03$ & $0,20^{\mathrm{a} b} \pm 0,01$ & $0,14^{\mathrm{b}} \pm 0,20$ & $0,22^{a, c} \pm 0,14$ \\
\hline $22: 4 n-6$ & $0,52^{\mathrm{a}} \pm 0,02$ & $0,52^{\mathrm{a}} \pm 0,03$ & $0,46^{\mathrm{a}} \pm 0,04$ & $0,46^{a} \pm 0,39$ \\
\hline $22: 5 n-6$ & $0,41^{\mathrm{a}} \pm 0,10$ & $0,49^{\mathrm{a}} \pm 0,03$ & $0,39^{\mathrm{a}} \pm 0,05$ & $0,40^{\mathrm{a}} \pm 0,15$ \\
\hline $22: 5 n-3$ & $0,21^{\mathrm{a}} \pm 0,01$ & $0,24^{\mathrm{a}} \pm 0,03$ & $0,29^{\mathrm{a}} \pm 0,03$ & $0,26^{\mathrm{a}} \pm 0,05$ \\
\hline $22: 6 n-3$ & $0,20^{\mathrm{b}} \pm 0,06$ & $0,29^{a} \pm 0,04$ & $0,32^{\mathrm{a}} \pm 0,04$ & $0,29^{a} \pm 0,05$ \\
\hline
\end{tabular}

Os resultados são médias com estimativas dos desvios padrão.

Resultados expressos em percentagem do total de ácidos graxos.

Letras diferentes na mesma linha indicam diferenças significativas $(p<0,05)$ pelo teste de Tukey

Nos lipídios totais das cabeças de tilápias, foram encontrados um total de 27 ácidos graxos. Os resultados percentuais de ácidos graxos mostram que os ácidos graxos majoritários em ordem decrescente, para um mesmo tratamento, foram: o ácido 18:2n-6, o 18:1n-9, seguido pelo 16:0, para os tratamentos $\mathrm{A}, \mathrm{B}, \mathrm{C}$, enquanto que no tratamento $\mathrm{D}$, houve uma inversão entre os ácidos 18:2n-6 e 18:1n-9, conforme mostra a Tabela 4. Apesar de não existirem diferenças significativas entre os tratamentos, a percentagem de 18:2n-6 foi sendo reduzida à medida que o tempo de alimentação com óleo de linhaça foi aumentando, do tratamento A para o D. Isto justifica a inversão do ácido 18:1n-9 com o 18:2n-6. Possivelmente, com um tempo de alimentação prolongada (acima de 30 dias) os niveis de 18:2n-6 seriam ainda mais reduzidos.

VISENTAINER et al.[29] encontraram os mesmos ácidos graxos majoritários em cabeças de tilápias jovens alimentadas com ração comercial, porém a ordem foi alterada $18: 1 n-9(30,86 \%), 16: 0(19,53 \%)$ e 18:2n-6 (18,76\%). Em filés de tilápias adultas alimentadas com ração comercial, também foram encontrados estes ácidos como majoritários, porém em ordem decrescente os resultados foram: 16:0 (28,9\%), 18:1n-9 (28,2) e 18:2n-6 (13,4\%) [15].

Foi observado (Tabela 4) que o fornecimento da ração à base de óleo de linhaça está diretamente relacionado à incorporação de ácidos graxos da família n-3 na cabeça das tilápias, isto ficou bem estabelecido na composição do ácido 18:3n-3 (LNA), 22:6n-3 (DHA) e na somatória de n-3 ( $\sum$ n-3), onde os valores foram superiores e com diferença $(\mathrm{P}<0,05)$ entre o tratamento $A$ e os tratamentos B, C e D.

Os teores do ácido $\alpha$-linolênico (LNA, 18:3n-3) apresentaram percentagem que variaram de 2,04 a 3,80\%, nos tratamentos (B, C, e D). Estes valores foram superiores aos encontrados por MOREIRA [19] em cabeças de espécies adultas cultivadas de Brycon (matrinxã, piraputanga e piracanjuba) com valores que variaram entre estas espécies de 0,51 a 0,97\%. Estas espécies de Brycon foram alimentadas com rações comerciais que apresentavam baixos teores de LNA. No entanto, os teores do ácido docosa-hexaenóico (DHA 22:6n-3), entre 0,20 e $0,32 \%$, destas tilápias (Tabela 4), foram inferiores às espécies de Brycon cultivadas, com valores de DHA que variaram de 0,37 a 1,60\%.

A Tabela 5 mostra as somatórias dos ácidos graxos: saturados (AGS), monoinsaturados (AGMI), polinsaturados (AGPI), ômega-3 (n-3) e ômega-6 (n-6); e as razões: somatória dos ácidos graxos polinsaturados/ somatória dos ácidos graxos saturados (AGPI/AGS) e somatória dos ácidos graxos ômega-6/somatória dos ácidos graxos ômega-3 (n-6/n-3).

Não foram encontradas diferenças $(P>0,05)$ entre os tratamentos (A, B, C e D) nas somatórias dos ácidos graxos saturados (AGS), polinsaturados (AGPI) e dos ácidos ômega-6 (n-6), conforme mostra a Tabela 5.

Do ponto de vista nutricional, resultados de pesquisas mostraram que a ingestão de ácidos graxos saturados aumenta os niveis de colesterol sérico em humanos [21] e que, os niveis de colesterol total no plasma sangüineo diminuem quando a ingestão de ácidos graxos saturados foi substituída por monoinsaturados [7]. 
TABELA 5. Somatórias e razões de grupos de ácidos graxos nos lipídios totais de cabeças de tilápias submetidas a diferentes tratamentos com óleo de linhaça.

\begin{tabular}{|c|c|c|c|c|}
\hline \multirow{2}{*}{$\begin{array}{l}\text { SOMATÓRIAS } \\
\text { e RAZÕES }\end{array}$} & \multicolumn{4}{|c|}{ TRATAMENTOS (em número de dias) } \\
\hline & $\mathrm{A}(0$ dias $)$ & $\mathrm{B}$ (10 dias) & $\mathrm{C}(20$ dias $)$ & $\mathrm{D}$ (30 dias) \\
\hline$\overline{\Sigma A G S}$ & $23,85^{\mathrm{a}} \pm 0,44$ & $24,65^{\mathrm{a}} \pm 0,97$ & $23,89^{\mathrm{a}} \pm 0,88$ & $24,30^{\mathrm{a}} \pm 1,78$ \\
\hline$\Sigma$ AGMI & $38,15^{\mathrm{a}} \pm 0,61$ & $36,16^{\mathrm{b}} \pm 0,76$ & $36,83^{\mathrm{a}, \mathrm{b}} \pm 1,48$ & $37,74^{\mathrm{a}} \pm 1,38$ \\
\hline$\Sigma$ AGPI & $37,99^{\mathrm{a}} \pm 1,05$ & $39,19^{\mathrm{a}} \pm 1,07$ & $39,28^{\mathrm{a}} \pm 0,59$ & $37,96^{\mathrm{a}} \pm 2,36$ \\
\hline$\Sigma \mathrm{n}-6$ & $34,65^{\mathrm{a}} \pm 0,92$ & $34,64^{\mathrm{a}} \pm 0,98$ & $33,06^{\mathrm{a}} \pm 0,55$ & $31,89^{\mathrm{a}} \pm 2,20$ \\
\hline$\Sigma \mathrm{n}-3$ & $1,71^{\mathrm{c}} \pm 0,14$ & $2,89^{\mathrm{b}} \pm 0,32$ & $4,74^{\mathrm{a}} \pm 0,15$ & $4,88^{\mathrm{a}} \pm 0,80$ \\
\hline$n-6 / 1-3$ & $20,27^{\mathfrak{c}} \pm 1,70$ & $11,98^{\mathrm{b}} \pm 1,37$ & $6,98^{\mathrm{a}} \pm 0,25$ & $6,53^{\mathrm{a}} \pm 1,17$ \\
\hline AGPI/AGS & $1,59^{a} \pm 0,05$ & $1,59^{\mathrm{a}} \pm 0,08$ & $1,64^{\mathrm{a}} \pm 0,07$ & $1,56^{\mathrm{a}} \pm 0,15$ \\
\hline
\end{tabular}

Os resultados são médias com estimativas dos desvios padrão. Resultados expressos em percentagem do total de ácidos graxo Letras diferentes na mesma linha indicam diferenças significativas $(p<0,05)$ pelo
teste de Tukey.

A somatória de AGS para os diferentes tratamentos foi de: $23,85 \%$ (A), 24,65\% (B), 23,89\% (C) e 24,30\% (D). Estes valores são inferiores aos encontrados em cabeças de Brycon (matrinxã, piraputanga e piracanjuba), cuja somatória de AGS variou de 40,29 a $45,61 \%$ [19] e também inferiores ao valor de 35,3\% encontrados por HENDERSON et al. [11] em cérebros de piranhas carnivoras.

Os valores das somatórias de AGMI variaram de 36,16 a $38,15 \%$ entre os tratamentos. Apesar de existirem diferenças significativas entre alguns tratamentos, não foram observadas diferenças bem estabelecidas nas somatórias de AGMI à medida que foi aumentado o período de alimentação com óleo de linhaça. Os valores das somatórias de AGMI foram encontrados em cabeças de várias espécies: 45,77\% em tilápias adultas [30]; $41,35 \%$ em tilápias jovens [29], 46,39\% matrinxã, $49,82 \%$ piraputanga e $48,26 \%$ piracanjuba [19].

Os AGS e AGMI nos peixes podem ter suas origens em função da dieta lipídica. Além da dieta, os ácidos saturados podem ser gerados pela sintese de novo [10] (utilizando fontes de carbono não lipídicas) e transformados em ácidos graxos monoinsaturados. E, ainda, a sintese de novo é inversamente proporcional aos niveis de saturados recebidos pela dieta [10]. Desta forma, os niveis destes ácidos nas diferentes espécies estão regidos por variáveis, como características individuais das espécies, tipo de alimentação, influência do meio onde sobrevivem, dentre outras.

Quanto à questão nutricional para humanos, ENSER et al. [8], baseados em informações do Departamento de Saúde da Inglaterra para alimentos integrais, recomendam que a razão n- $6 /$ n-3 seja no máximo 4,0. Entretanto, não há consenso entre os pesquisadores quanto à ingestão de ácidos graxos w6 e w3. SIMOPOULOS et al. [25] recomendam um intervalo entre 5 e 10 para a razão n-6/n-3.

Neste trabalho, as razões n- $6 / \mathrm{n}-3$ nos lipídios totais foram: A $(20,27)$, B $(11,98), \mathrm{C}(6,98)$ e D $(6,53)$. As razões foram decrescentes do tratamento $A$ para $D$ e com diferenças $(\mathrm{P}<0,05)$ entre alguns tratamentos, especialmente entre o tratamento $\mathrm{A}$ e os demais tratamentos. Isto mostra que, o tempo de alimentação das tilápias com óleo de linhaça contribuiu decisivamente para reduzir os valores da razão n-6/n-3 e melhorar acentuadamente o valor nutricional da composição lipídica das cabeças de tilápias.

A comparação dos valores das razões $n-6 / n-3$ das cabeças de tilápias deste experimento, tratamento $\mathrm{C}$ $(6,98)$ e $D(6,53)$, com as razões n-6/n-3 das cabeças de piracanjuba $(7,23)$, matrinxã $(8,04)$ e piraputanga $(6,59)$ [19] mostram que, todas estas espécies apresentaram valores da razão n6/n3 dentro do intervalo de 5 e 10, conforme recomendado por SIMOPOULOS et al. [25]. Todavia, as tilápias dos tratamentos C e D apresentaram razões n6/n3 próximos ao valor máximo de 4,0, recomendado pelo Departamento de Saúde da Inglaterra, segundo ENSER et al. [8].

Os valores das razões n- $6 / \mathrm{n}-3$ em filés de tilápias: 14,1 [15], 30,32 [13] e, em filés de pacu 14,3 [17] e tambaqui 9,8 [16] foram superiores aos valores encontrados para o tratamento $\mathrm{C}(6,98)$ e $\mathrm{D}(6,53)$ deste experimento.

Para a razão das somatórias de AGPI/AGS, o Departamento de Saúde da Inglaterra, citado por ELSER et al. [8], argumenta que razões inferiores a 0,45 indicam carnes pouco saudáveis, especialmente com relação às doenças cardiovasculares. Neste sentido, alimentos com maiores valores AGPI/AGS indicam valor nutricional superior.

Neste experimento, as razões de AGPI/AGS para os diferentes tratamentos foram: A $(1,59), \mathrm{B}(1,59), \mathrm{C}(1,64)$ e $\mathrm{D}(1,56)$, sem diferenças $(\mathrm{P}<0,05)$ entre os tratamentos. Os valores foram superiores a 0,45 , conforme recomendação do Departamento de Saúde da Inglaterra, citado por ELSER et al. [8].

Alguns autores encontraram razões de AGPI/AGS em cabeças de espécies de matrinxã $(0,33)$ e piraputanga $(0,39)$ [19], em filés de tilápias $(0,2)[15]$, pacu $(0,1)$ [17] e tambaqui $(0,10)[16]$. Estas razões indicam que estas cabeças e filés constituem alimentos pouco saudáveis, pois todos os valores foram inferiores a 0,45 . Neste sentido, estas cabeças e filés apresentaram valor nutricional inferior ao encontrado nas cabeças de tilápias de todos os tratamentos (A, B, C e D), deste experimento.

\section{4 - CONCLUSÕES}

- A composição em ácidos graxos das dietas, assim como o seu tempo de fornecimento, foram refletidos diretamente na composição de ácidos graxos das cabeças de tilápias (Oreochromis niloticus).

- O aumento dos teores percentuais de ácidos graxos da família ômega-3 foi estabelecido, à medida que o tempo de alimentação com óleo de linhaça foi aumentando.

- Apesar das cabeças de tilápias não apresentarem elevados niveis de ácidos graxos ômega-3, mesmo 
aquelas que receberam óleo de linhaça por 20 ou 30 dias, as mesmas constituem uma boa fonte destes ácidos, devido ao elevado teor de lipídios totais existente na cabeça, o qual foi superior a muitos outros peixes.

- As cabeças de tilápias, submetidas aos tratamentos com rações à base de óleo de linhaça deste experimento, apresentaram valor nutricional superior às cabeças e filés de outras espécies de peixes. Assim, elas podem ser utilizadas na alimentação humana, como uma fonte de ácidos graxos ômega3 , de elevado conteúdo calórico e de baixo custo.

- Contudo, ainda há necessidade de estudos de viabilidade econômica para a utilização de cabeças de tilápias deste experimento, como matéria-prima para a produção de concentrados de ômega-3.

\section{5 - REFERÊNCIAS BIBLIOGRÁFICAS}

[1] ANDRADE, A. D.; RUBIRA, A. F.; MATSUSHITA, M.; SOUZA, N. E. w-3 Fatty acids in freshwater fish from south Brazil. Journal of American Oil Chemists Society, v. 72, p. 1207-10, 1995.

[2] BLIGH, E. G.; DYER, W. J. A rapid method of total lipid extraction and purification. Canadian Journal of Biochemistry, v. 37, p. 911-17, 1959.

[3] CARTER, J. F. Potential of flaxseed and flaxseed oil in baked goods and other products in human nutrition. Cereal Foods world, v. 38, p. 753-59, 1993.

[4] CEOTTO, B. O que é que a linhaça tem. Dentro das sementes da planta que dá origem ao linho há componentes que equilibram os hormônios femininos e reforçam as defesas do corpo. Revista Saúde, p. 37-40, Jan/2000.

[5] CONQUER, J. A.; MARTIN, J. B.; TUMMON, I.; WATSON, L.; TEKPETEY, F. Effect of DHA supplementation on DHA status and sperm motility in asthenozoospermic males. Lipids, v. 35, p. 149-54, 2000.

[6] CUNNIF, P. A. (Ed.) Official Methods of Analysis of AOAC international. 16th ed. Arlington: Association of Official Analytical Chemists. 1998. CD-Rom.

[7] DEPARTMENT OF HEALTH. Nutritional aspects of cardiovascular disease. Report on health and social subjects. n. 46 HMSO, London, 1994.

[8] ENSER, M.; HALLETT, K. G.; FURSEY, A. J.; WOOD, J. D.; HARRINGTON, G. Fatty acid content and composition of UK beef and lamb muscle in relation to production system and implications for human nutrition. Meat Science, v. 49, n. 3, p. 329-341, 1998.

[9] HARRIS, W.S. Nonpharmacologic treatment of hypertriglyceridemia: focus on fish oils. Clinical Cardiology, v. 22, (suppl. II): p. 40-3, 1999.

[10] HENDERSON, R. J. Fatty acid metabolism in freshwater fish with particular reference to polyunsaturated fatty acids. Archives of Animal Nutrition, v. 49, p. 5-22, 1996.

[11] HENDERSON, R. J.; TILLMANNS, M. M.; SARGENT, J. R. The lipid composition of two species of Serrasalmid fish in relation to dietary polyunsaturated fatty acids. Journal of Fish Biology, v. 48, p. 522-538, 1996.

[12] ISO - INTERNATIONAL ORGANIZATION FOR STANDARDIZATION. Animal and vegetable fats and oils - preparation of methyl esters of fatty acids. Geneve: ISO. Method ISO 5509, p. 01-06, 1978.
[13] JUSTI, K.C. Composição físico-química e ácidos graxos em tilápias do nilo (Oreochromis niloticus) submetidas a dietas e temperaturas diferenciadas. 2001. Dissertação de Mestrado. Departamento de Química, Universidade Estadual de Maringá. Maringá, PR.

[14] KINSELLA, J. E. Food components with potential therapeutic benefits: the n-3 polyunsaturated fatty acids of fish oils. Food Technology, v. 40, p. 89-97, 1986.

[15] MAIA, E. L. Otimização de metodologia para caracterização de constituintes lipídicos e determinação da composição em ácidos graxos e aminoácidos de peixes de água doce. 1992. Tese de Doutorado. FEA/Unicamp, Campinas, SP.

[16] MAIA, E. L.; RODRIGUEZ-AMAYA, D. B. Fatty acid composition of the total, neutral and phospholipids of the Brazilian freshwater fish Colossoma macropomum. In Food Science and Human Nutrition (G. Charalambous, Ed.), p. 633-42. Elsevier Science, Amsterdam, 1992.

[17] MAIA, E. L.; RODRIGUEZ-AMAYA, D. B.; HOTTA, L. K. Fatty acids composition of the total, neutral and phospholipids of pond-raised Brazilian Piaractus mesopotamicus. International Journal of Food Science and Technology. v. 30, 591-97, 1995.

[18] MAYER, P. ; MROWIETZ, U.; ARENBERGER, P. ; BARTAK, P. BUCHVALD, J.; CHRISTOPHERS. E.; JABLONSKA, S. SALMHOFER, W.; SCHILL, W. B.; KRAMER, H. J.; SCHLOTZER, E. MAYER, K.; SEEGER, W. GRIMMINGER, F. Omega-3 fatty acid-based lipid infusion in patients with chronic plaque psoriasis: results of a double-blind, randomized, placebo-controlled, multicenter trial. J. Am. Acad. Dermatol., v. 38, p. 421, 1998.

[19] MOREIRA, A. B. Caracterização fĩsico química e perfil dos ácidos graxos de três espécies de Brycon. 2001. Dissertação de Mestrado. Departamento de Química, Universidade Estadual de Maringá, Maringá, PR.

[20] OLIVEIRA, E. R. N. Composição química geral e de ácidos graxos da fração lipídica de peixes do reservatório de Itaipu, Paraná-Brasil: Relação com variáveis biológicas e período de coleta. 2000. Tese de doutorado. UEM, Maringá, PR.

[21] SANCHES-MUÑIZ, F. J.; CUESTA, C. Lipid metabolism in experimental animals. Grasas y Aceites. v. 49, p. 3406, 1998.

[22] SIGUEL, E. A new relationship between total/high density lipoprotein cholesterol and polyunsaturated fatty acids. Lipids, v. 31, p. 51-6, 1996.

[23] SILVA, A. J. I. Composição lipídica e quantificação dos ácidos graxos polinsaturados EPA (20:5 n-3) e DHA (22:6 n-3) de peixes de água doce. 2000. Tese de doutorado. UNICAMP, Campinas - SP.

[24] SIMOPOULOS, A. P. Omega-3 fatty acids in health and disease and in growth and development. Am. J. Clin. Nutr., v. 54, p. 438-63, 1991.

[25] SIMOPOULOS, A. P. ; LEAF, A.; SALEM, N. Essentiality and recommended dietary intakes for omega- 6 and omega-3 fatty acids. Annals of Nutrition and Metabolism., v. 43, p. 127-30, 1999.

[26] STATISTICA (1995). Statistica 5.1 Software. Tucksa: StaSoft.

[27] UAUY, R.; VAlEnZUELA, A. Marine oils: The health benefits of $\mathrm{n}-3$ fatty acids. Nutrition, v. 16, n. $7 / 8$, p. 680-84, 2000.

[28] VISENTAINER, J. V.; CARVALHO, P. ' ; IKEGAKI, M.; PARK, Y. K. Concentração de ácido eicosapentaenóico (EPA) e ácido docosahexaenóico (DHA) em peixes marinhos da 
costa brasileira. Ciênc. Tecnol. Aliment., v. 20, n. 1, p. 90-93, 2000.

[29] VISENTAINER, J.V.; HAYASHI, C.; SOARES, C.M.; GALDIOLI, E.M; FRANCO, M.R.B. Quantificação de ácidos graxos ômega-3 (LNA, Epa e DHA), caracterização físico química e composição de ácidos graxos em cabeças de tilápias jovens. XII Congresso Brasileiro de Ciência e Tecnologia de Alimentos, Fortaleza, CE. p. 5.221, 2000.

[30] VISENTAINER, J.V.; SOUZA, D.B.; FRANCO, M.R.B. Quantificação de ácidos graxos ômega-3 e composição de ácidos graxos em cabeças de tilápias submetidas à die- ta prolongada. IV Simpósio Latino Americano de Ciência e Tecnologia de Alimentos, Campinas, SP. p. $72,2001$.

[31] WEAVER, B. J.; HOLOB, B. J. Health effects and metabolism of dietary eicosapentaenoic acid. Progress in food and nutrition Science, v. 12, p. 111-50, 1998.

\section{6 - AGRADECIMENTOS}

Os autores agradecem ao CNPq e CAPES pelo aporte financeiro. 